\title{
Bio Efficacy of Different Novel Insecticides against Cotton Thrips, T. tabaci in Transgenic Cotton
}

\author{
Ramalakshmi, Lipsa Dash", Deepayan Padhy and G. M. V. Prasada Rao \\ MSSSoA, Centurion University of Technology and Management, Odisha, India \\ *Corresponding author
}

\section{A B S T R A C T}

\section{Keywords}

Cotton thrips , insecticides, efficacy

\section{Article Info}

Accepted:

05 April 2020

Available Online:

10 May 2020
To assess the efficacy of different insecticides for the management of cotton Thrips, an experiment was conducted at Regional Agricultural Research Station, Lam, Guntur during 2011-12. Among the different tested insecticides Fipronil 5\% SC@ 50g.a.i/ha has shown 76.7per cent reduction of thrips, followed by Fipronil 80\% WG@ 50g.a.i/ha, Acephate 75\% SP@750g.a.i/ha and Imidacloprid 70\% WG@21g.a.i/ha has shown 74.5, 71.6 and 69.0per cent reduction over the control after ten days after treatment. Furthermore, it has recorded highest yield of $13.5 \mathrm{q} / \mathrm{ha}$ when compared to other treatments.

\section{Introduction}

Cotton is an important fibre crop of global significance cultivated in more than seventy countries. It is an important raw material for the Indian textile industry and plays a key role in the national economy in terms of both employment generation and foreign exchange. India thus enjoys the distinction of being the earliest country in the world to domesticate and to utilize its fibre to manufacture fabric (Mayee et al., 2004). In India cotton ecosystem harbours about 162 insect pest species and the monetary value of estimated yield losses due to insect pests has been estimated to be Rs 3,39,660 million annually (Dhaliwal et al., 2010). Among the sap feeders aphids Aphis gossypii (Glover), Leafhoppers Amrascabiguttula biguttula (Ishida), thrips Thrips tabaci (Linn) and whitefly Bemisia tabaci are deadly pests. A Cotton grower in India depends heavily on synthetic pesticides to combat sucking pests.

Atleast2-3 sprays are directed against sucking pests. Due to continuous and indiscriminate use of synthetic insecticides, there is resistance and hence the efficacy has become 
less reliable. To overcome this problem discovery of novel substances with different biochemical targets are needed.

A number of broad-spectrum insecticides, with a comparatively longer residual effect, are being sprayed, as a common practice by the farmers. In India, at least 2-3 sprays are directed against the sucking pests (Acharya et $a l ., 2002)$. This practice wipes off the useful fauna from the field and leads to complex pest problem and flare up of one or other pest, in such situation, there is every need to suggest more selective insecticide, which have less deleterious effects on the beneficials

Several potent insecticides have been recommended for managing sucking pests, but the use of insecticides have resulted in the development of resistance, resurgence, secondary pest out breaks, disruption of natural enemy complex and environmental pollution (Dhaliwal and Arora, 2001).

The newer molecules have a higher stability and superiority over the conventional insecticides to control the pest population density at field level (Vinoth Kumar et al., 2009). Fipronil 5\% SC @ 50-75 gm a.i. ha ${ }^{-1}$ dose was found optimum against aphids, leafhoppers and thrips of cotton (Wadnerkar et al., 2003). Imidacloprid70 WG @ 40 g a.i. $\mathrm{ha}^{-1}$ provided good protection against aphids, thrips, whiteflies and leafhoppers of cotton (Naveen et al., 2010). The investigation was therefore under taken for the suitable management practices to combat the thrips damage.

\section{Materials and Methods}

The experiment was laid out in Randomized Block Design with ten treatments including control and replicated thrice with plot size of $6.3 \mathrm{~m} \mathrm{X} 5.4 \mathrm{~m}$. Standard agronomic practices were adopted to raise a good crop of cotton.
$B t$ cotton hybrid RCH-2BG-II was selected for this experiment. Treatment particulars are presented in table-1.

\section{Seed treatment}

For delinted seed, $5 \mathrm{ml}$ of gum per $\mathrm{kg}$ seed was evenly distributed through thorough shaking in a polythene bag into which $5 \mathrm{~g}$ of imidacloprid 70 WS was added for uniform coating over the seed. Then the treated seed was shade dried for about 10 minutes and used for sowing.

\section{Application of treatments}

A measured quantity of insecticidal solution /powder was mixed with a little quantity of water and stirred well, after which the remaining quantity of water was added to obtain the required concentration of spray fluid. Sprayings were given by using a hand compression knapsack high volume sprayer, during morning hours.

The plot in each treatment was sprayed with respective insecticides ensuring uniform coverage of insecticide. The sprayer and the accessories were thoroughly washed before changing the insecticides and also rinsed with the spray fluid of the chemical to be applied next. The first spraying was given at 60 DAS when the incidence of sucking pest population was sufficiently built up in the experimental plots. A total of three sprays were given during the course of season at ten days interval.

\section{Recording observations}

The incidence of sucking pests viz., aphids, leafhoppers, whiteflies and thrips were recorded by counting the number of nymphs and adults per three leaves, per plant on five randomly selected plants per plot at 3, 7and 10 days after treatment. 
The seed cotton yield from each plot was recorded twice separately in $\mathrm{kg} / \mathrm{plot}$ and converted into $\mathrm{q} / \mathrm{ha}$

\section{Results and Discussion}

\section{Mean efficacy of different novel insecticides against thrips}

Mean data on thrips at 3 DAT ranged from 3.2 to $16.6 /$ three leaves presented in table(Table-2, 3, Fig. 1). . Fipronil 5\% SC @ 50 g a.i.ha ${ }^{-1}(3.2$ / three leaves) was the most effective treatment followed by fipronil $80 \%$ WG @ 50 g a.i. ha ${ }^{-1}$ (3.5/ three leaves) and acephate 75\% SP @ $750 \mathrm{~g}$ a.i. ha ${ }^{-1}$ (4.0/ three leaves) which were on par with each other.

The next best treatments; imidacloprid $70 \%$ WG @21 g a.i. ha $^{-1}$ (4.4/three leaves), spirotetramat150 OD @ 90 g a.i. ha ${ }^{-1}(4.7 /$ three leaves), spiromesfin 240 SC @ 40 g a.i. ha $^{-1}$ (5.0/three leaves) and thiacloprid $21.7 \%$ SC @ $24 \mathrm{~g}$ a.i. ha $^{-1}(5.5 /$ three leaves $)$.The treatments, diafenthiuron 50\% WP @ $375 \mathrm{~g}$ a.i. $h^{-1}(6.3 /$ three leaves) and buprofezin $25 \%$ SC @ 150 g a.i. ha ${ }^{-1}(6.8 /$ three leaves $)$ were on par with each other and significantly superior over untreated control.

Similar trend was observed at 7 and 10 DAT. At 7DAT population ranged from 3.5to17.0/three leaves. Fipronil 5\% SC @ 50 $\mathrm{g}$ a.i.ha ${ }^{-1}$ (3.5 / three leaves) was the effective treatment followed by fipronil 80\% WG @ $50 \mathrm{~g}$ a.i. $\mathrm{ha}^{-1}(3.8 /$ three leaves $)$ and acephate 75\% SP @ 750 g a.i. ha ${ }^{-1}$ (4.3/ three leaves).

The next best treatments were imidacloprid $70 \%$ WG (4.6/three leaves), spirotetramat 150 OD @ $90 \quad \mathrm{~g} \quad$ a.i. ha $^{-1}$ (5.1/three leaves),spiromesfin 240 SC @ $40 \mathrm{~g}$ a.i. ha ${ }^{1}$ (5.5/ three leaves), thiacloprid 21.7\% SC @ $24 \mathrm{~g}$ a.i. $\mathrm{ha}^{-1}$ (6.1/three leaves) .The treatments, diafenthiuron 50\% WP @ $375 \mathrm{~g}$ a.i. $\mathrm{ha}^{-1}(6.9 /$ three leaves $)$ and buprofezin
25\% SC@150 g a.i. ha ${ }^{-1}$ (7.3/three leaves) were on par with each other and superior over untreated control.

The thrips population ranged from 4.0 to17.5/ three leaves at 10DAT. Fipronil 5\% SC @ 50 g a.i.ha ${ }^{-1}$ (4.0/three leaves) was the most effective treatment followed by fipronil $80 \%$ WG @ 50 g a.i. ha ${ }^{-1}$ (4.4/ three leaves) and acephate $75 \%$ SP @ 750 g a.i. ha ${ }^{-1}$ (4.9/three leaves).

The next best treatments were imidacloprid 70\%WG @21 g a.i. ha ${ }^{-1}$ (5.3/three leaves), spirotetramat 150 OD @ 90 g a.i. ha 15.7/three leaves),spiromesfin 240 SC @ 40 $\mathrm{g}$ a.i. $\mathrm{ha}^{-1}(6.1 /$ three leaves $)$ and thiacloprid $21.7 \%$ SC @ 24 g a.i. ha ${ }^{-1}$ (6.8/three leaves) .The treatments, diafenthiuron 50\% WP @ $375 \mathrm{~g}$ a.i. $\mathrm{ha}^{-1}(7.4 /$ three leaves $)$ and buprofezin 25\% $\quad$ SC @ 150 g a.i. ha $^{-1}$ (8.0/three leaves) were on par with each other and significantly superior over untreated control.

Per cent reduction of thrips population at 10 DAT, indicated highest reduction in fipronil 5\% SC @ 50 g a.i. ha ${ }^{-1}(76.7 \%)$ followed by fipronil 80\% WG @ $50 \mathrm{~g}$ a.i. ha ${ }^{-1}(74.5 \%)$ and acephate $75 \%$ SP @ 750 g a.i. ha ${ }^{-1}$ $(71.6 \%)$. The next best treatments were imidacloprid 70\% WG @21 g a.i. ha ${ }^{1}(69.0 \%)$, spirotetramat 150 OD @ 90 g a.i. $\mathrm{ha}^{-1}(67.0 \%)$, spiromesfin 240 SC @ $40 \mathrm{~g}$ a.i. $\mathrm{ha}^{-1}(64.7 \%)$ and thiacloprid $21.7 \%$ SC @ $24 \mathrm{~g}$ a.i. $\mathrm{ha}^{-1}(60.8 \%)$.The treatments, d iafenthiuron 50\% WP @ $375 \mathrm{~g}$ a.i. ha ${ }^{-1}$ and buprofezin 25\% SC @150 g a.i. ha ${ }^{-1}$ recorded reduction of $57.5 \%$ and $54.3 \%$ respectivly.

Per cent reduction in observed during first, second and third spray at 10DAT was $64.1 \%$, $76.7 \%$ and $88.6 \%$ respectively. These findings conformity with that Mau et al., (1998) reported that fipronil @ 0.01\% was highly effective against $T$. tabaci infesting 
onion. Kadam and Dethe (2002) findings revealed that fipronil $5 \mathrm{SC}$ at the rate of 40 to $60 \mathrm{~g}$ a.i. $\mathrm{ha}^{-1}$, when applied as a schedule of four sprays at an interval of 15 days by initiating the first spray 4 weeks after transplanting, was effective in lowering the thrips count to 3.32-9.63 as against a count of 13.44-23.43 in untreated control in chilli.

Rupal and Dethe (2002) reported that four sprays of fipronil 5 SC @ 40 -60 g a.i. ha ${ }^{-1}$ gave $91.2 \%$ mortality of $S$. dorsalis in chilli. Jadhav et al., (2004) indicated that fipronil 5 SC @ 100 g a.i. ha $^{-1}$ resulted in 2.2 leafhoppers per leaf and 1.2 thrips per leaf at seven days after application in chilli.

Ghosh et al., (2009) reported that fipronil 5 SC @ $75 \mathrm{~g}$ a.i. ha ${ }^{-1}$ gave $88.8 \%$ mortality of S. dorsalis in chilli. Patil et al., (2009) recorded that fipronil 5\% SC @ 800g/ ha registered least number of thrips (8.47 / 3 leaves) and significantly highest seed cotton yield of $27.23 \mathrm{q} / \mathrm{ha}$ (2007) and $27.50 \mathrm{q} / \mathrm{ha}$
(2008) was harvested. Information of fipronil agents cotton thrips is limited.however these findings corroborate with findings on thrips of other crops like onion and chilli. (Rohini, 2010) reported that fipronil $5 \mathrm{SC}$ at $0.01 \%$ effective against thrips population. Fipronil $5 \% \mathrm{SC}$ recorded least number of thrips 3.51 per three leaves in cotton (Zanwar et al., 2012)

The next best treatments were acephate $75 \%$ SP @ $750 \mathrm{~g}$ a.i. $\mathrm{ha}^{-1}$ and Imidacloprid 70\% WG @ $21 \mathrm{~g}$ a.i. ha ${ }^{-1}$ The present findings are in agreement with Ameta and Sharma (2005) who reported that imidacloprid $70 \mathrm{WG}$ at $35 \mathrm{~g}$ a.i. $\mathrm{ha}^{-1}$ caused the highest reduction in population of thrips in cotton at $1,3,5$ and 7 days after first and second sprays. Wahla et al., (1997) reported that Confidor 200 SL at $40 \mathrm{ml} / \mathrm{acre}$ was the most effective against cotton thrips. Saleem et al., (2001) reported that Confidor200 SL effectively controlled thrips up to seven days after the spray in cotton.

Table.1 Particulars of insecticides used

\begin{tabular}{|c|l|l|c|}
\hline S.No. & \multicolumn{1}{|c|}{ Chemical name } & \multicolumn{1}{|c|}{ Chemical class } & a.i. ha $^{-1}$ \\
\hline $\mathbf{T}_{\mathbf{1}}$ & Diafenthiuron 50\% WP & Thiourea & 375 \\
\hline $\mathbf{T}_{\mathbf{2}}$ & Fipronil 5\%SC & Phenylpyrazole & 50 \\
\hline $\mathbf{T}_{\mathbf{3}}$ & Spirotetramat150 OD & Ketoenols & 90 \\
\hline $\mathbf{T}_{\mathbf{4}}$ & Imidacloprid 70\% WG & Neonicotinoids & 21 \\
\hline $\mathbf{T}_{\mathbf{5}}$ & Fipronil 80\% WG & Phenylpyrazole & 50 \\
\hline $\mathbf{T}_{\mathbf{6}}$ & Buprofezin 25\% SC & Insect growth regulator & 150 \\
\hline $\mathbf{T}_{\mathbf{7}}$ & Spiromesifen 240 SC & Spirocyclictetronic acids & 40 \\
\hline $\mathbf{T}_{\mathbf{8}}$ & Thiacloprid 21.7\% SC & Neonicotinoids & 24 \\
\hline $\mathbf{T}_{\mathbf{9}}$ & Acephate 75\% SP & Organophosphate & 750 \\
\hline
\end{tabular}


Table.2 Bioefficacy of different novel insecticides against thrips, T.tabaci

\begin{tabular}{|c|c|c|c|c|c|c|c|c|c|c|c|c|c|}
\hline \multirow{2}{*}{ S.NO } & \multirow{2}{*}{ Treatments } & \multicolumn{4}{|c|}{ First spray } & \multicolumn{4}{|c|}{ Second spray } & \multicolumn{4}{|c|}{ Third spray } \\
\hline & & 3DAT* & 7DAT* & 10DAT* & $\begin{array}{l}\% \text { reduction } \\
\text { over control } \\
\text { at 10DAT** }\end{array}$ & 3DAT* & 7DAT* & 10DAT* & $\begin{array}{l}\% \text { reduction } \\
\text { over control } \\
\text { at } 10 \mathrm{DAT} * *\end{array}$ & 3DAT* & 7DAT* & 10DAT* & $\begin{array}{l}\% \text { reduction } \\
\text { over control } \\
\text { at } 10 \mathrm{DAT} * *\end{array}$ \\
\hline T1 & Diafenthurion50\%WP & $\begin{array}{c}7.9 \\
(2.99)^{\mathrm{b}}\end{array}$ & $\begin{array}{c}8.2 \\
(3.03)^{\mathrm{bc}}\end{array}$ & $\begin{array}{c}8.7 \\
(3.12)^{\mathrm{ab}}\end{array}$ & $\begin{array}{c}47.5 \\
(43.61)^{\mathrm{a}}\end{array}$ & $\begin{array}{c}6.1 \\
(2.66)^{\mathrm{b}}\end{array}$ & $\begin{array}{c}7.3 \\
(2.89)^{\mathrm{b}}\end{array}$ & $\begin{array}{c}7.7 \\
(2.94)^{\mathrm{b}}\end{array}$ & $\begin{array}{c}56.0 \\
(47.4)^{\mathrm{bc}}\end{array}$ & $\begin{array}{c}4.8 \\
(2.41)^{\mathrm{c}}\end{array}$ & $\begin{array}{c}5.1 \\
(2.46)^{\mathrm{cd}}\end{array}$ & $\begin{array}{c}5.7 \\
(2.58)^{\mathrm{ef}}\end{array}$ & $\begin{array}{c}69.0 \\
(56.19)^{\mathrm{ef}}\end{array}$ \\
\hline T2 & Fipronil $5 \%$ SC & $\begin{array}{c}5.1 \\
(2.46)^{\mathrm{a}}\end{array}$ & $\begin{array}{c}5.4 \\
(2.53)^{\mathrm{a}}\end{array}$ & $\begin{array}{c}5.9 \\
(2.63)^{\mathrm{a}}\end{array}$ & $\begin{array}{c}64.1 \\
(53.28)^{\mathrm{a}}\end{array}$ & $\begin{array}{c}3.3 \\
(2.07)^{\mathrm{a}}\end{array}$ & $\begin{array}{c}3.5 \\
(2.13)^{\mathrm{a}}\end{array}$ & $\begin{array}{c}4.0 \\
(2.24)^{\mathrm{a}}\end{array}$ & $\begin{array}{c}76.7 \\
(61.3)^{\mathrm{a}}\end{array}$ & $\begin{array}{c}1.4 \\
(1.55)^{\mathrm{a}}\end{array}$ & $\begin{array}{c}1.6 \\
(1.61)^{\mathrm{a}}\end{array}$ & $\begin{array}{c}2.1 \\
(1.75)^{\mathrm{a}}\end{array}$ & $\begin{array}{c}88.6 \\
(70.31)^{\mathrm{a}}\end{array}$ \\
\hline $\mathbf{T 3}$ & Spirotetramat 150 OD & $\begin{array}{c}6.5 \\
(2.73)^{\mathrm{ab}}\end{array}$ & $\begin{array}{c}6.9 \\
(2.80)^{\mathrm{abc}}\end{array}$ & $\begin{array}{c}7.6 \\
(2.93)^{\mathrm{ab}}\end{array}$ & $\begin{array}{c}54.2 \\
(47.42)^{\mathrm{a}}\end{array}$ & $\begin{array}{c}4.9 \\
(2.42)^{\mathrm{ab}}\end{array}$ & $\begin{array}{c}5.3 \\
(2.52)^{\mathrm{ab}}\end{array}$ & $\begin{array}{c}5.6 \\
(2.57)^{\mathrm{ab}}\end{array}$ & $\begin{array}{c}67.9 \\
(55.5)^{\mathrm{ab}}\end{array}$ & $\begin{array}{c}2.8 \\
(1.95)^{\mathrm{b}}\end{array}$ & $\begin{array}{c}3.1 \\
(2.02)^{\mathrm{b}}\end{array}$ & $\begin{array}{c}3.9 \\
(2.21)^{\mathrm{bcd}}\end{array}$ & $\begin{array}{c}78.5 \\
(62.43)^{\text {bcd }}\end{array}$ \\
\hline T4 & Imidacloprid 70\% WG & $\begin{array}{c}6.1 \\
(2.67)^{\mathrm{ab}}\end{array}$ & $\begin{array}{c}6.4 \\
(2.72)^{\mathrm{abc}}\end{array}$ & $\begin{array}{c}7.2 \\
(2.86)^{\mathrm{ab}}\end{array}$ & $\begin{array}{c}55.4 \\
(48.25)^{\mathrm{a}}\end{array}$ & $\begin{array}{c}4.5 \\
(2.34)^{\mathrm{ab}}\end{array}$ & $\begin{array}{c}4.9 \\
(2.42)^{\mathrm{ab}}\end{array}$ & $\begin{array}{c}5.3 \\
(2.52)^{\mathrm{ab}}\end{array}$ & $\begin{array}{c}69.0 \\
(56.2)^{\mathrm{ab}}\end{array}$ & $\begin{array}{c}2.5 \\
(1.88)^{\mathrm{b}}\end{array}$ & $\begin{array}{c}2.7 \\
(1.91)^{\mathrm{b}}\end{array}$ & $\begin{array}{c}3.5 \\
(2.11)^{\mathrm{abcd}}\end{array}$ & $\begin{array}{c}81.1 \\
(64.24)^{\mathrm{abcd}}\end{array}$ \\
\hline T5 & Fipronil $80 \%$ WG & $\begin{array}{c}5.3 \\
(2.50)^{\mathrm{a}}\end{array}$ & $\begin{array}{c}5.7 \\
(2.58)^{\mathrm{ab}}\end{array}$ & $\begin{array}{c}6.4 \\
(2.72)^{\mathrm{ab}}\end{array}$ & $\begin{array}{c}60.6 \\
(51.27)^{\mathrm{a}}\end{array}$ & $\begin{array}{c}3.7 \\
(2.16)^{\mathrm{ab}}\end{array}$ & $\begin{array}{c}3.9 \\
(2.21)^{\mathrm{ab}}\end{array}$ & $\begin{array}{c}4.2 \\
(2.28)^{\mathrm{a}}\end{array}$ & $\begin{array}{c}75.5 \\
(60.4)^{\mathrm{a}}\end{array}$ & $\begin{array}{c}1.5 \\
(1.59)^{\mathrm{ab}}\end{array}$ & $\begin{array}{c}1.7 \\
(1.65)^{\mathrm{ab}}\end{array}$ & $\begin{array}{c}2.5 \\
(1.88)^{\mathrm{ab}}\end{array}$ & $\begin{array}{c}86.3 \\
(68.27)^{\mathrm{ab}}\end{array}$ \\
\hline T6 & Buprofezin $25 \%$ SC & $\begin{array}{c}8.4 \\
(3.07)^{\mathrm{b}}\end{array}$ & $\begin{array}{c}8.8 \\
(3.13)^{\mathrm{c}}\end{array}$ & $\begin{array}{c}9.4 \\
(3.22)^{\mathrm{b}}\end{array}$ & $\begin{array}{c}43.2 \\
(41.09)^{\mathrm{b}}\end{array}$ & $\begin{array}{c}6.6 \\
(2.76)^{\mathrm{b}}\end{array}$ & $\begin{array}{c}7.5 \\
(2.91)^{\mathrm{b}}\end{array}$ & $\begin{array}{c}8.0 \\
(3.00)^{\mathrm{b}}\end{array}$ & $\begin{array}{c}54.28 \\
(47.48)^{\mathrm{c}}\end{array}$ & $\begin{array}{c}5.3 \\
(2.52)^{\mathrm{c}}\end{array}$ & $\begin{array}{c}5.5 \\
(2.54)^{\mathrm{d}}\end{array}$ & $\begin{array}{c}6.6 \\
(2.76)^{\mathrm{f}}\end{array}$ & $\begin{array}{c}63.8 \\
(53.01)^{\mathrm{f}}\end{array}$ \\
\hline $\mathbf{T 7}$ & Spiromesfin $240 \mathrm{SC}$ & $\begin{array}{c}6.9 \\
(2.80)^{\mathrm{ab}}\end{array}$ & $\begin{array}{c}7.3 \\
(2.89)^{\mathrm{abc}}\end{array}$ & $\begin{array}{c}7.9 \\
(2.98)^{\mathrm{ab}}\end{array}$ & $\begin{array}{c}52.7 \\
(46.54)^{\mathrm{a}}\end{array}$ & $\begin{array}{c}5.3 \\
(2.50)^{\mathrm{ab}}\end{array}$ & $\begin{array}{c}5.9 \\
(2.62)^{\mathrm{b}}\end{array}$ & $\begin{array}{c}6.3 \\
(2.70)^{\mathrm{b}}\end{array}$ & $\begin{array}{c}62.3 \\
(52.4)^{\mathrm{abc}}\end{array}$ & $\begin{array}{c}3.0 \\
(2.00)^{\mathrm{b}}\end{array}$ & $\begin{array}{c}3.4 \\
(2.10)^{\mathrm{bc}}\end{array}$ & $\begin{array}{c}4.5 \\
(2.27)^{\text {cde }}\end{array}$ & $\begin{array}{c}75.4 \\
(60.03)^{\mathrm{cde}}\end{array}$ \\
\hline T8 & Thiacloprid $21.7 \%$ SC & $\begin{array}{c}7.3 \\
(2.88)^{\mathrm{ab}}\end{array}$ & $\begin{array}{c}7.7 \\
(2.96)^{\mathrm{abc}}\end{array}$ & $\begin{array}{c}8.2 \\
(3.03)^{\mathrm{ab}}\end{array}$ & $\begin{array}{c}50.3 \\
(45.19)^{\mathrm{a}}\end{array}$ & $\begin{array}{c}5.7 \\
(2.59)^{\mathrm{b}}\end{array}$ & $\begin{array}{c}6.8 \\
(2.79)^{\mathrm{b}}\end{array}$ & $\begin{array}{c}7.3 \\
(2.88)^{\mathrm{b}}\end{array}$ & $\begin{array}{c}56.0 \\
(48.6)^{\mathrm{bc}}\end{array}$ & $\begin{array}{c}3.5 \\
(2.13)^{\mathrm{bc}}\end{array}$ & $\begin{array}{c}3.9 \\
(2.21)^{\mathrm{c}}\end{array}$ & $\begin{array}{c}4.9 \\
(2.42)^{\mathrm{def}}\end{array}$ & $\begin{array}{c}73.5 \\
(59.02)^{\text {def }}\end{array}$ \\
\hline T9 & Acephate $75 \%$ SP & $\begin{array}{c}5.7 \\
(2.58)^{\mathrm{ab}}\end{array}$ & $\begin{array}{c}6.1 \\
(2.67)^{\mathrm{abc}}\end{array}$ & $\begin{array}{c}6.8 \\
(2.79)^{\mathrm{ab}}\end{array}$ & $\begin{array}{c}58.9 \\
(50.17)^{\mathrm{a}}\end{array}$ & $\begin{array}{c}4.1 \\
(2.27)^{\mathrm{ab}}\end{array}$ & $\begin{array}{c}4.5 \\
(2.34)^{\mathrm{ab}}\end{array}$ & $\begin{array}{c}4.8 \\
(2.41)^{\mathrm{ab}}\end{array}$ & $\begin{array}{c}73.5 \\
(59.3)^{\mathrm{a}}\end{array}$ & $\begin{array}{c}2.1 \\
(1.77)^{\mathrm{ab}}\end{array}$ & $\begin{array}{c}2.4 \\
(1.84)^{\mathrm{ab}}\end{array}$ & $\begin{array}{c}3.1 \\
(2.02)^{\mathrm{abc}}\end{array}$ & $\begin{array}{c}83.1 \\
(65.76)^{\mathrm{abc}}\end{array}$ \\
\hline T10 & Control (untreated) & $\begin{array}{c}15.1 \\
(4.02)^{\mathrm{c}}\end{array}$ & $\begin{array}{c}15.9 \\
(4.12)^{\mathrm{d}}\end{array}$ & $\begin{array}{c}16.5 \\
(4.19)^{\mathrm{c}}\end{array}$ & & $\begin{array}{c}16.9 \\
(4.23)^{\mathrm{c}}\end{array}$ & $\begin{array}{c}17.3 \\
(4.28)^{\mathrm{c}}\end{array}$ & $\begin{array}{c}17.5 \\
(4.30)^{\mathrm{c}}\end{array}$ & & $\begin{array}{c}17.7 \\
(4.32)^{\mathrm{d}}\end{array}$ & $\begin{array}{c}17.9 \\
(4.35)^{\mathrm{e}}\end{array}$ & $\begin{array}{c}18.3 \\
(4.40)^{\mathrm{g}}\end{array}$ & \\
\hline & F-TEST & Sig & sig & sig & sig & Sig & sig & Sig & sig & sig & sig & sig & Sig \\
\hline & SEm & 0.14 & 0.14 & 0.17 & 3.58 & 0.17 & 0.15 & 0.15 & 3.13 & 0.10 & 0.09 & 0.11 & 1.13 \\
\hline & $\mathrm{CD}(\mathrm{P}=\mathbf{0 . 0 5})$ & 0.43 & 0.43 & 0.52 & 10.6 & 0.51 & 0.45 & 0.45 & 9.30 & 0.30 & 0.28 & 0.34 & 5.84 \\
\hline *Figur & parentheses are s & coot tra & ormed & des. & & Sig & $: \mathrm{S}$ & ficant. & & & & & \\
\hline$* *$ Figu & in parentheses are a & $\operatorname{ar}$ transf & med va & & & NS & $.1 \mathrm{v}$ & signific & & & & & \\
\hline Numbe & ollowed by same su & cript are & ot statist & ally diffe & & $\mathrm{DA}$ & $: \mathrm{Da}$ & after tre & ment & & & & \\
\hline
\end{tabular}


Table.3 Mean efficacy of different novel insecticides against thrips, T.tabaci

\begin{tabular}{|c|c|c|c|c|c|}
\hline S.NO & Treatments & 3DAT* & 7DAT* & 10DAT* & $\begin{array}{l}\% \text { reduction over } \\
\text { control at 10DAT } * *\end{array}$ \\
\hline T1 & Diafenthurion50\%WP & $\begin{array}{l}6.3 \\
(2.69)^{\mathrm{cd}}\end{array}$ & $\begin{array}{l}6.9 \\
(2.79)^{\mathrm{ef}}\end{array}$ & $\begin{array}{l}7.4 \\
(2.88)^{\mathrm{ef}}\end{array}$ & $\begin{array}{l}57.5 \\
(49.41)^{\mathrm{ef}}\end{array}$ \\
\hline $\mathbf{T} 2$ & Fipronil $\quad 5 \%$ SC & $\begin{array}{l}3.2 \\
(2.03)^{\mathrm{a}}\end{array}$ & $\begin{array}{l}3.5 \\
(2.09)^{\mathrm{a}}\end{array}$ & $\begin{array}{l}4.0 \\
(2.21)^{\mathrm{a}}\end{array}$ & $\begin{array}{l}76.7 \\
(61.70)^{\mathrm{a}}\end{array}$ \\
\hline T3 & Spirotetramat 150 OD & $\begin{array}{l}4.7 \\
(2.37)^{\mathrm{abc}}\end{array}$ & $\begin{array}{l}5.1 \\
(2.45)^{\mathrm{bcd}}\end{array}$ & $\begin{array}{l}5.7 \\
(2.57)^{\mathrm{bcd}}\end{array}$ & $\begin{array}{l}67.0 \\
(55.20)^{\mathrm{cd}}\end{array}$ \\
\hline $\mathbf{T 4}$ & Imidacloprid $70 \% \mathrm{WG}$ & $\begin{array}{l}4.4 \\
(2.30)^{\mathrm{ab}}\end{array}$ & $\begin{array}{l}4.6 \\
(2.35)^{\mathrm{abcd}}\end{array}$ & $\begin{array}{l}5.3 \\
(2.50)^{\mathrm{abc}}\end{array}$ & $\begin{array}{l}69.0 \\
(56.50)^{\mathrm{bc}}\end{array}$ \\
\hline T5 & Fipronil $80 \%$ WG & $\begin{array}{l}3.5 \\
(2.09)^{\mathrm{a}}\end{array}$ & $\begin{array}{l}3.8 \\
(2.15)^{\mathrm{ab}}\end{array}$ & $\begin{array}{l}4.4 \\
(2.29)^{\mathrm{ab}}\end{array}$ & $\begin{array}{l}74.5 \\
(60.15)^{\mathrm{ab}}\end{array}$ \\
\hline T6 & Buprofezin $25 \% \mathrm{SC}$ & $\begin{array}{l}6.8 \\
(2.78)^{d}\end{array}$ & $\begin{array}{l}7.3 \\
(2.86)^{\mathrm{f}}\end{array}$ & $\begin{array}{l}8.0 \\
(2.99)^{\mathrm{f}}\end{array}$ & $\begin{array}{l}54.3 \\
(47.48)^{\mathrm{f}}\end{array}$ \\
\hline $\mathbf{T 7}$ & Spiromesfin $240 \%$ SC & $\begin{array}{l}5.0 \\
(2.44)^{\mathrm{bc}}\end{array}$ & $\begin{array}{l}5.5 \\
(2.53)^{\text {cde }}\end{array}$ & $\begin{array}{l}6.1 \\
(2.65)^{\text {cde }}\end{array}$ & $\begin{array}{l}64.7 \\
(53.79)^{\text {cde }}\end{array}$ \\
\hline T8 & Thiacloprid $21.7 \%$ SC & $\begin{array}{l}5.5 \\
(2.53)^{\mathrm{bcd}}\end{array}$ & $\begin{array}{l}6.1 \\
(2.65)^{\text {def }}\end{array}$ & $\begin{array}{l}6.8 \\
(2.78)^{\operatorname{def}}\end{array}$ & $\begin{array}{l}60.8 \\
(51.39)^{\mathrm{de}}\end{array}$ \\
\hline T9 & Acephate $75 \%$ SP & $\begin{array}{l}4.0 \\
(2.21)^{\mathrm{ab}}\end{array}$ & $\begin{array}{l}4.3 \\
(2.28)^{\mathrm{abc}}\end{array}$ & $\begin{array}{l}4.9 \\
(2.41)^{\mathrm{abc}}\end{array}$ & $\begin{array}{l}71.6 \\
(58.16)^{\mathrm{abc}}\end{array}$ \\
\hline \multirow[t]{4}{*}{ T10 } & $\begin{array}{c}\text { Control } \\
\text { (untreated) }\end{array}$ & $\begin{array}{l}16.6 \\
(4.19)^{\mathrm{e}}\end{array}$ & $\begin{array}{l}17.0 \\
(4.25)^{\mathrm{g}}\end{array}$ & $\begin{array}{l}17.5 \\
(4.30)^{\mathrm{g}}\end{array}$ & \\
\hline & F-TEST & sig & Sig & sig & sig \\
\hline & SEm & 0.06 & 0.05 & 0.05 & 0.85 \\
\hline & $\mathrm{CD}(\mathrm{P}=0.05)$ & 0.31 & 0.29 & 0.27 & 4.41 \\
\hline $\begin{array}{l}\text { * Figure } \\
\text { **Figure } \\
\text { Numbers }\end{array}$ & $\begin{array}{l}\text { arentheses are square root trat } \\
\text { oarentheses are angular transf } \\
\text { owed by same superscript are }\end{array}$ & $\begin{array}{l}\text { rmed values. } \\
\text { ed values. } \\
\text { statistically diff }\end{array}$ & & $\begin{array}{l}\text { : Signif } \\
\text { : Non-s } \\
\quad \text { : Days }\end{array}$ & $\begin{array}{l}\text { ant. } \\
\text { nificant. } \\
\text { ter treatment }\end{array}$ \\
\hline
\end{tabular}

Table.4 Seed cotton yield

\begin{tabular}{|c|c|c|}
\hline S.NO & Treatments & YIELD(q/ha) \\
\hline T1 & Diafenthurion50\%WP & 12.7 \\
\hline T2 & Fipronil 5\% SC & 13.5 \\
\hline T3 & Spirotetramat 150 OD & 9.3 \\
\hline T4 & Imidacloprid 70\% WG & 11.1 \\
\hline T5 & Fipronil 80\% WG & 13.4 \\
\hline T6 & Buprofezin25\%SC & 12.2 \\
\hline T7 & Spiromesfin 240 SC & 10.1 \\
\hline T8 & Thiacloprid 21.7\% SC & 8.6 \\
\hline T9 & Acephate 75\% SP & 11.4 \\
\hline T10 & Control (untreated) & 7.2 \\
\hline & F-TEST & sig \\
\hline & SEm & $\mathbf{0 . 4 0}$ \\
\hline & CD(P=0.05) & $\mathbf{2 . 0 7}$ \\
\hline
\end{tabular}




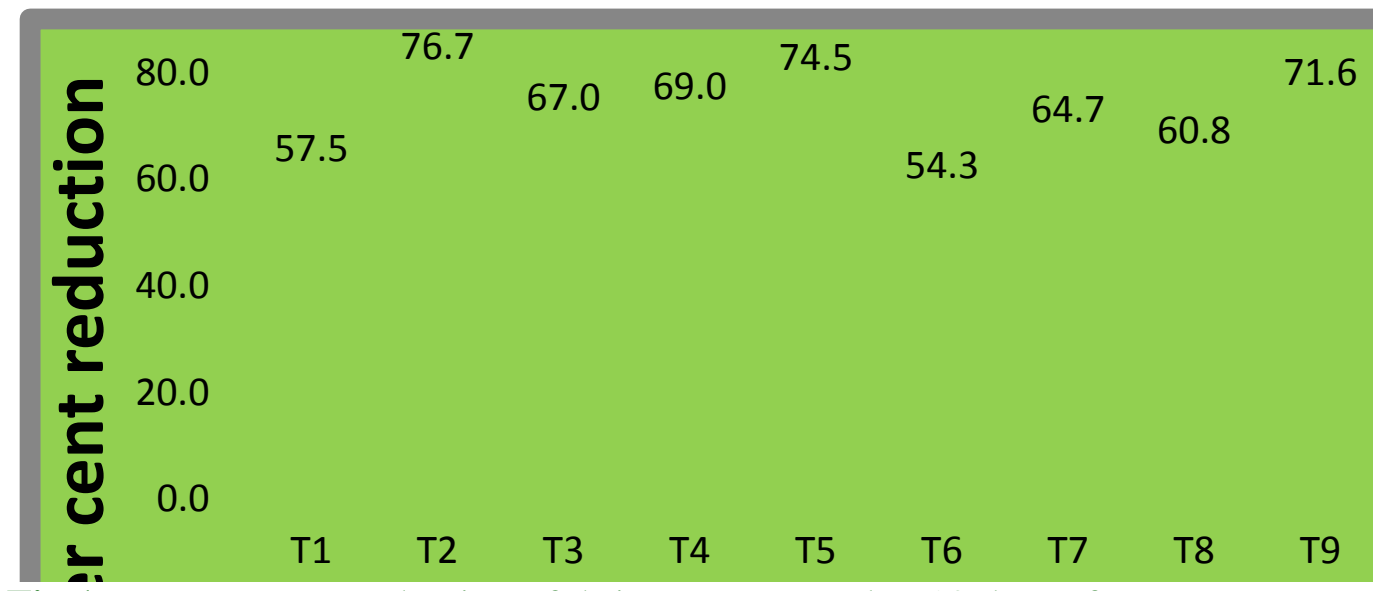

Fig.1 Mean per cent reduction of thrips over control at 10 days after treatment

$\begin{array}{lll}\text { T1:Diafenthiuron 50\% } & \text { WP } & -375 \text { ga.i. } \mathrm{ha}^{-1} \\ \text { T3: Spirotetramat 150\% } & \text { OD } & -90 \text { ga.i. } \mathrm{ha}^{-1} \\ \text { T5: Fipronil } 80 \% & \text { WG } & -50 \text { ga.i. } \mathrm{ha}^{-1} \\ \text { T7: Spiromesfin 240\% } & \text { SC } & -40 \text { ga.i. } \mathrm{ha}^{-1} \\ \text { T9: Acephate75\% } & \text { SP } & -750 \text { ga.i. ha }\end{array}$

Overall in the management of cotton thrips, Fipronil 5\% SC @ 50g.a.i/ha andFipronil 80\% WG @ 50g.a.i/haare superior over other treatments.

\section{References}

Acharya, S., Mishra, H.P and Dash, D. 2002. Efficacy of insecticides against Okra jassid, A.biguttulabiguttula. Annals of Plant Protection Sciences 10: 230-232.

Ameta, O. P and Sharma, K.C. 2005. Evaluation of Confidor for the management of sucking insect pests of cotton.Pestology. 29(2):35-40.

Dhaliwal, G.S and Arora, R. 2001. Role of phytochemicals in integrated pest management. In: phytochemical biopesticides, Koul, $\mathrm{O}$ and Dhaliwal, G.S (Eds), Harwood Academic publishers, Amsterdam, The Netherland. 97-117.

Dhaliwal, G.S and Vikas Jindal and Dhawan, A.K. 2010.insect pest problems and crop losses: changing trends. Indian Journal of Entomology. 37(1):1-7.

$\begin{array}{lll}\text { T2: Fipronil 5\% } & \text { SC } & -50 \text { ga.i. } \mathrm{ha}^{-1} \\ \text { T4: Imidacloprid 70\% } & \text { WG } & -21 \mathrm{~g} \text { a.i. } \mathrm{ha}^{-1} \\ \text { T6:Buprofezin 25\% } & \text { SC } & -150 \mathrm{~g} \text { a.i. } \mathrm{ha}^{-1} \\ \text { T8:Thiacloprid 21.7\% } & \text { SC } & -24 \text { a.i. ha }\end{array}$

Ghosh, A., Chatterjee, M. L., Chakrabotri, K and Samanta, A. 2009.Field evaluation of insecticides against chillithrips (Scirtothrips dorsalis Hood).Annals of Plant Protection Sciences. 17(1):69-71.

Jadhav, V. R., Wadnerkar, D. W and Jayewar, N. E. 2004.Fipronil 5\% SC: An effective insecticide against sucking pets of chilli (Capsicum annum Linn). Pestology. 28(10):84-87.

Kadam, R. V and Dethe, M. D. 2002. Fipronil formulations for effective control of chillithrips, Scirtothrips dorsalis (Hood). Pestology. 26(4):36-38.

Mau, R..F.L., Gusukuma, M. L., Vierbergen, $\mathrm{G}$ and Tunccedilla, I.D. 1998. Insecticidal management of key pests of fruiting vegetables, onions and corn in Hawaii.Proceedings of Sixth International Symposium on Thysanoptera, Akdeniz University, Antalya, Turkey. April 27- May 18:107112.

Mayee, C.D., Gautam, H.C and Barik, A. 2004.Cotton scenario in India vis-a-vis world and future need. In: Recent 
Advances in Cotton Research andDevelopment. (Chauhan, M.S. and.Sain, R.K., Eds.).Haryana Agricultural University and Cotton Research and Development Association, CCSHAU, Hisar. PP 245 - 253.

Naveen.A.,Vikas, J and Vikram, S. 2010. Comparative efficacy of insecticides against sucking pests complex in transgenic cotton. Pestology.34(8): 45-49.

Patil, S.B., Udikeri, S.S., Matti, P.V., Guruprasad, G.S., Hirekurumbar, R.B., Saila, H.M and Vandal,N.B. 2009. Bioefficacy of new molecule fipronil5\%SC against sucking pest complex in Btcotton. Karnataka Journal of Agricultural Sciences. 22(5): 1029-1031.

Rohini, A.2010.Screening of germplasm and evaluation of insecticides for the management of major sucking pests on cotton (Gossypiumhirsutum L.M. Sc Thesis. Acharya N.G Ranga Agricultural University, Hyderabad.

Rupal, V. K and Dethe, M. D. 2002.Fipronil formulations for effective control of chillithrips, Scirtothrips dorsalis
(Hood). Pestology. 26(4):36-38.

Saleem, M.A., Khalid, M and Riaz, H. 2001. Comparative efficacy of some insecticides against some sucking insect pests of CIM-443, cotton.Pakistan Entomology. 23(1/2):91-92.

VinothKumar, B., Kuttalam, S and Chandrasekaran. 2009. Efficacy of a new insecticide spirotetramat against cotton whitefly. Pesticide Research Journal. 21(1): 45-48.

Wadnerkar, D. W., Kawthekar, B. R and Zanwar, P. R. 2003. Evaluation of fipronil 5\% SC against cotton insect pests.Pestology .27(9):15-18.

Wahla, M. A., Tufail, M and Parvez, I. 1997. The comparative effectiveness of different doses of Confidor SL 200 and Tamaron 600 SL against the cotton thripsThripstabaci Lind.On FH-682, cotton.Pakistan Entomologist. 19(1/2):8-10.

Zanwar, P.R., Deosarkar, D.B., Yadav, G.A and Shelke, L.T. 2012. Evaluation of certain neonicotinoids against sucking pests in Bt cotton. Pestology. 36(1):2124.

\section{How to cite this article:}

Ramalakshmi, Lipsa Dash, Deepayan Padhy and Prasada Rao, G. M. V. 2020. Bio Efficacy of Different Novel Insecticides against Cotton Thrips, T. tabaci in Transgenic Cotton. Int.J.Curr.Microbiol.App.Sci. 9(05): 88-95. doi: https://doi.org/10.20546/ijcmas.2020.905.008 